\title{
Standard Logics Are Valuation-Nonmonotonic
}

\author{
Mladen Pavičić \\ Physics Chair, Faculty of Civil Engineering \\ University of Zagreb, Zagreb, Croatia \\ pavicic@grad.hr; http://m3k.grad.hr/pavicic \\ Norman D. Megill \\ Boston Information Group, 19 Locke Ln. \\ Lexington, MA 02420, USA \\ nm@alum.mit.edu; http://www.metamath.org
}

June 3, 2018

\begin{abstract}
It has recently been discovered that both quantum and classical propositional logics can be modelled by classes of non-orthomodular and thus non-distributive lattices that properly contain standard orthomodular and Boolean classes, respectively. In this paper we prove that these logics are complete even for those classes of the former lattices from which the standard orthomodular lattices and Boolean algebras are excluded. We also show that neither quantum nor classical computers can be founded on the latter models. It follows that logics are valuation-nonmonotonic in the sense that their possible models (corresponding to their possible hardware implementations) and the valuations for them drastically change when we add new conditions to their defining conditions. These valuations can even be completely separated by putting them into disjoint lattice classes by a technique presented in the paper.

Keywords: nonmonotonic logic, classical logic, quantum logic, nondistributive non-orthomodular lattice, weakly orthomodular lattice, Boolean algebra, weakly distributive lattice, artificial intelligence
\end{abstract}




\section{Introduction}

A good deal of artificial intelligence research is focused on artificial neural networks, on the one hand, and on default/nonmonotonic logic, on the other. Neural networks are characterized by heavy reliance on logic gates. On the other hand, nonmonotonic inference rules formalize generalizations of standard logic that admit changes in the sense that values of propositions may change when new information (axioms) is added to or old information is deleted from the system. In this paper, we show that already standard logics (classical as well as quantum) - whose monotonicity is usually taken for granted - are nonmonotonic at both the level of logic gates that implement them and the level of its valuations, i.e., mappings from the logic to its models.

We consider two standard logics (in contrast to, e.g., modal logics) in this paper: propositional classical logic and propositional quantum logic. In practice, classical logic relies almost exclusively on the $\{0,1\}$ valuation, i.e., the two-valued truth table valuation, for its propositional part. This valuation extends to the sentences of all theories that make use of classical logic, such as set theory, model theory, and the foundations of mathematics. However, there are also non-standard valuations generated by non-distributive lattices, which correctly model classical propositional logic, and by non-orthomodular lattices, which correctly model quantum logic. An immediate consequence of this valuation dichotomy is that classical logic modelled by such nondistributive lattices does not underlie present-day classical computers, since non-standard valuations cannot be used to run them. Only classical logic modelled by a Boolean algebra and having a $\{0,1\}$ valuation can serve us for such a purpose. Hence, whenever we want to utilize a logic for a particular application we have to specify the model we would use as well.

Before we go into details in the next sections, we should be more specific about our distinction of standard vs. non-standard valuations. Let us illustrate it with a graphical representation of the O6 lattice given in Figure 1, which can serve as a model for classical logic in the same way that $\{0,1\}$ Boolean algebra can. Lines in the figure mean ordering. Thus we have $0 \leq x \leq y \leq 1$ and $0 \leq y^{\prime} \leq x^{\prime} \leq 1$, where 0 and 1 are the least and the greatest elements of the lattice, respectively. Can this model be given a linearly ordered or numerical interpretation, for instance the interpretation provided by the probabilistic semantics for classical logic [1]? The answer is no, because when $x \neq y \neq 0,1$, an ordering between $x$ and either $x^{\prime}$ or $y^{\prime}$ and 
between $y$ and either $x^{\prime}$ or $y^{\prime}$ is not defined, and it is assumed that it cannot be defined. Hence, symbols 1 and 0 in the figure cannot be interpreted as the numbers 1 and 0 . If they were numbers, $0<x<y<1$ and $0<y^{\prime}<x^{\prime}<1$ would imply that $x, y$ and $x^{\prime}, y^{\prime}$ were also numbers and we would, for example have $x=0.3$ and $x^{\prime}=0.7$. This means we would have $x<x^{\prime}$ and it yields $x \cap x<x^{\prime} \cap x=0$, i.e., $x=0$, which is a contradiction, since $x \neq 0$.

Therefore when we speak of standard valuation of propositions of classical logic, we mean any valuation for which we can establish a correspondence with real numbers and their ordering, i.e., whose corresponding model can be totally ordered. For instance, with two-valued ( $\{$ TRUE,FALSE $\}$ ) Boolean algebra we can ascribe the number 1 to TRUE and the number 0 to FALSE, and in the probabilistic interpretation of classical logic [1 all values from the interval $[0,1]$ are real numbers which are totally ordered. When we deal with values from our O6 example above, there is no way to establish a correspondence of $\mathrm{O} 6$ elements with real numbers, and we shall call such a valuation non-standard. The point here is that the latter valuation cannot be implemented in present-day binary computers - whose hardware usually deals with numerical values such as voltage - and consequently also not in the corresponding artificial intelligence, at the level of the underlying logic gates building their hardware.

This means that a statement from a logic can be "true" or "false" in one model in one way and in some other model in another way. When it "holds" (i.e., is "true") in a standard model, say the two-valued Boolean algebra, we can ascribe a number to it, say "1". When it "holds" in a non-standard model, meaning, e.g., that it is equal to 1 in Figure 1, we cannot do so and we cannot evaluate the model for the statement directly with binary logic gates.

It is usually taken for granted that logic is about propositions and their values. For example, we are tempted to assume that proposition $p$ meaning "Material point $q$ is at position $\mathbf{r}$ at time $t$ " is either true or false. However, with non-standard valuations $x$ and $y$ from Figure 1, we can ascribe neither a truth value nor even a probability to $p$, although " $p$ or non- $p$ " is certainly always valid meaning $p \cup p^{\prime}=1$. The $\{0,1\}$ Boolean algebra and the probabilistic model, on the other hand, are the only known classical logical models that allow ascribing $\{0,1\}$ standard (i.e., numerical) values to propositions and hence "found[ing] the mathematical theories of logic and probabilities" [2]. Classical logic defined by nothing but its axiomatic syntax is a more general theory, in terms of the possible valuations it may have, than 
its non-isomorphic semantics (e.g., a predicate logical calculus with standard valuation 1 which is nothing but a "predicate Boolean algebra").

The standard-non-standard dichotomy can be even better understood with the example of quantum logic which - when taken together with its orthomodular lattice model-underlies Hilbert space and therefore could be implemented into would-be quantum computers and eventually into quantum artificial intelligence. According to the Kochen-Specker theorem, a $\{0,1\}$ valuation for quantum logic does not exist.2 but there is an analogy between a Boolean algebra (distributive ortholattice) and an orthomodular (ortho)lattice that underlies the Hilbert space of quantum mechanics. Every orthomodular lattice is a model of quantum logic just as every Boolean algebra (distributive ortholattice) is a model of classical logic. However, as with classical logic, there are also non-orthomodular lattices which are models of quantum logic but on which no Hilbert space can be built. Therefore quantum logic in general (not modelled by any model, i.e., without any semantics), or more precisely its syntax, would be of limited use if we wanted to implement it into quantum computers. Only one of its models - an orthomodular lattice - can serve us for this goal, and therefore we call valuations defined on the elements of the latter model-standard valuations, as opposed to non-standard valuations on the former non-orthomodular models.

In this paper, we prove the nonmonotonicity of both classical and quantum logic with respect to particular intrinsically different, disjoint classes of models. The result separates two kinds of models that have so far been assumed to belong to overlapping classes. In particular, general families of non-distributive and non-orthomodular lattices called weakly orthomodular and weakly distributive ortholattices (WOML and WDOL) that are models of quantum and classical propositional logics, respectively, for which we pre-

\footnotetext{
1 "A quantificational schema is valid if it comes out true under all interpretations in all nonempty universes... [T] he truth value of a compound statement depends on no features of the component sentences and terms except their truth values and their extensions... [Quantificational] schema [containing sentence letters] will be valid, clearly, just in case it resolves to ' $T$ ' or to a valid schema under each substitution of ' $T$ ' and ' $\perp$ ' for its sentence letters. So [its] test is truth-value analysis." [3 p. 131]

${ }^{2}$ In 2004 we gave exhaustive algorithms for generation of Kochen-Specker vector systems with arbitrary number of vectors in Hilbert spaces of arbitrary dimension. [4, 5, 6] The algorithms use MMP (McKay-Megill-Pavičić) diagrams for which in 3-dim Hilbert space a direct correspondence to Greechie and Hasse diagrams can be established. Thus, we also have a constructive proof of the non-existence of a $\{0,1\}$ valuation within the lattice itself.
} 
viously proved soundness and completeness [7, 8], do include their standard models, orthomodular lattices (OML) and Boolean algebras (BA) [distributive ortholattices (DOL)]. Here we prove that these lattices can be separated in the sense that the logics can also be modelled by WOML and WDOL from which the standard orthomodular and Boolean algebras are excluded $]^{3}$ Soundness and completeness of these propositional logics are proved.

Specifically, we consider the proper subclasses of these lattice families that exclude those lattices that are orthomodular (for the WOML case) and distributive (for the WDOL case), i.e., WOML $\backslash \mathrm{OML}$ and WDOL $\backslash \mathrm{BA}$ (where "Y" denotes set-theoretical difference). Using them as the basis for a modification of the standard Lindenbaum algebra technique, we present a new result showing that quantum and classical propositional logics are respectively complete for these proper subclasses, in and of themselves, as models. In other words, even after removing every lattice from WOML (WDOL) in which the orthomodular (distributive) law holds, quantum (classical) propositional logic is still complete for the remaining lattices.

In both classical and quantum logics, when we add new conditions to the defining conditions of the lattices that model the logics, we get new lattices that also model these logics but with changed valuations for the propositions from the logics. This property of standard logics and valuations of their propositions is what we call valuation-nonmonotonicity. The more conditions we add, the fewer choices we have for valuations. This is why we consider subclasses that exclude lattices obtained by adding new conditions. For instance, WOML $\backslash$ OML will provide us only with valuations on weakly orthomodular lattices that are not orthomodular, and by adding the orthomodularity condition to WOML we get OML, which contains only valuations on orthomodular lattices. Apart from the orthomodularity condition, there are many more (if not infinitely many) conditions in between WOML and OML that all provide different valuations and new proper subclasses, as we show and discuss in Sections 8 and 9 below.

We will study the quantum logic case first, since the results we obtain for WOMLs will automatically hold for WDOLs and simplify our subsequent presentation of the latter. In Section 2, we define orthomodular and weakly

\footnotetext{
${ }^{3}$ The names weakly orthomodular and weakly distributive ortholattices stem from the fact that in general these lattice families contain orthomodular and distributive ones, although in the light of the present "disjointness results" the names seem to be somewhat inappropriate. Recall also that at the beginning orthomodular lattices were called weakly modular lattices. [9]
} 
orthomodular (ortho)lattices, and in Section 3 distributive and weakly distributive ones. In Section 4, we define the classes of proper weakly orthomodular and proper weakly distributive ortholattices. In Section 5, we define quantum and classical logics and prove their soundness for the models defined in Section 4. In Sections 6 and 7, we prove the completeness of quantum logic for WOML $\backslash \mathrm{OML}$ and WDOL $\backslash \mathrm{BA}$ models respectively. In Section 8 , we define valuation-nonmonotonicity, and in Sections 8 and 9, we discuss the differences between the completeness proofs for WOML $\backslash$ OML, WDOL $\backslash$ BA, WOMLi $\backslash$ OML, WDOLi $\backslash \mathrm{BA}$, WOML $\backslash$ WOMLi, and WDOL $\backslash$ WDOLi we obtain in Sections 6.9 and the completeness proofs for WOML and WDOL we obtained in [7, 8]. And finally, we discuss and summarize the results we obtained in this paper in Section [10.

\section{Orthomodular and Weakly Orthomodular Lattices}

Definition 2.1 An ortholattice, OL, is an algebra $\left\langle\mathcal{O} \mathcal{L}_{0}{ }^{\prime}, \cup, \cap\right\rangle$ such that the following conditions are satisfied for any $a, b, c \in \mathcal{O} \mathcal{L}_{0}$ [10]:

$$
\begin{aligned}
& a \cup b=b \cup a \\
& (a \cup b) \cup c=a \cup(b \cup c) \\
& a^{\prime \prime}=a \\
& a \cup\left(b \cup b^{\prime}\right)=b \cup b^{\prime} \\
& a \cup(a \cap b)=a \\
& a \cap b=\left(a^{\prime} \cup b^{\prime}\right)^{\prime}
\end{aligned}
$$

In addition, since $a \cup a^{\prime}=b \cup b^{\prime}$ for any $a, b \in \mathcal{O} \mathcal{L}_{0}$, we define the greatest element of the lattice (1) and the least element of the lattice (0):

$$
1 \stackrel{\text { def }}{=} a \cup a^{\prime}, \quad 0 \stackrel{\text { def }}{=} \mathrm{a} \cap \mathrm{a}^{\prime}
$$

and the ordering relation $(\leq)$ on the lattice:

$$
a \leq b \quad \Longleftrightarrow \quad a \cap b=a \Longleftrightarrow a \cup b=b
$$


Connectives $\rightarrow_{1}($ Sasaki hook $), \rightarrow_{2}$ (Dishkant implication $), \rightarrow_{5}$ (relevance implication $), \rightarrow_{0}$ (classical implication), $\equiv$ (quantum equivalence), and $\equiv_{0}$ (classical equivalence) are defined as follows:

Definition 2.2 $a \rightarrow_{1} b \stackrel{\text { def }}{=} a^{\prime} \cup(a \cap b), \quad a \rightarrow_{2} b \stackrel{\text { def }}{=} b^{\prime} \rightarrow_{1} a^{\prime}$, $a \rightarrow_{5} b \stackrel{\text { def }}{=}(a \cap b) \cup\left(a^{\prime} \cap b\right) \cup\left(a^{\prime} \cap b^{\prime}\right), \quad a \rightarrow_{0} b \stackrel{\text { def }}{=} a^{\prime} \cup b$.

Definition 2.34 $\quad a \equiv b \stackrel{\text { def }}{=}(a \cap b) \cup\left(a^{\prime} \cap b^{\prime}\right)$.

Definition 2.4 $a \equiv_{0} b \stackrel{\text { def }}{=}\left(a \rightarrow_{0} b\right) \cap\left(b \rightarrow_{0} a\right)$.

Connectives bind from weakest to strongest in the order $\rightarrow_{1}\left(\rightarrow_{0}\right)$, 三 $\left(\equiv_{0}\right), \cup, \cap$, and ' .

Definition 2.5 If, in an ortholattice, $a=(a \cap b) \cup\left(a \cap b^{\prime}\right)$, we say that $a$ commutes with $b$, which we write as $a C b$.

Definition 2.6 If, in an ortholattice, $a \equiv\left((a \cap b) \cup\left(a \cap b^{\prime}\right)\right)=1$, we say that a weakly commutes with $b$, and we write this as $a C_{w} b$.

Definition 2.7 The commutator of $a$ and $b, C(a, b)$, is defined as $(a \cap b) \cup$ $\left(a \cap b^{\prime}\right) \cup\left(a^{\prime} \cap b\right) \cup\left(a^{\prime} \cap b^{\prime}\right)$.

Definition 2.8 (Pavičić and Megill [7]) An ortholattice in which the following condition holds:

$$
\left(a^{\prime} \cap(a \cup b)\right) \cup b^{\prime} \cup(a \cap b)=1
$$

is called a weakly orthomodular ortholattice (WOML).

Using Definition 2.2, we can also express Eq. (9) as either of the two following equations, which are equivalent in an ortholattice:

$$
\begin{aligned}
& \left(a \rightarrow_{2} b\right)^{\prime} \cup\left(a \rightarrow_{1} b\right)=1 \\
& \left(a \rightarrow_{1} b\right)^{\prime} \cup\left(a \rightarrow_{2} b\right)=1 .
\end{aligned}
$$

\footnotetext{
tice.

${ }^{4}$ In every orthomodular lattice $a \equiv b=\left(a \rightarrow_{1} b\right) \cap\left(b \rightarrow_{1} a\right)$, but not in every ortholat-
} 
Definition 2.9 An ortholattice in which either of the following conditions hold: [1]

$$
\begin{array}{r}
a \equiv b=1 \quad \Rightarrow \quad a=b \\
a \cup\left(a^{\prime} \cap(a \cup b)\right)=a \cup b
\end{array}
$$

is called an orthomodular lattice (OML).

The equations of Definition 2.1 determine a (proper) class of lattices, called an equational variety, [12, p. 352] that we designate OL. Thus the term OL will have two meanings, depending on context. When we say a lattice is an OL, we mean that the equations of Definition 2.1 hold in that lattice. When we say a lattice is in OL, we mean that it belongs to the equational variety OL determined by those equations. While these two statements are of course equivalent, the distinction will matter when we say such things as "the class OL properly includes the class OML." Similar remarks apply to OML, WOML, and the other varieties in this paper.

We recall that whereas every OML is a WOML, there are WOMLs that are not OMLs. [7] In particular, the lattice O6 (Fig. 1) is a WOML but is not an OML.

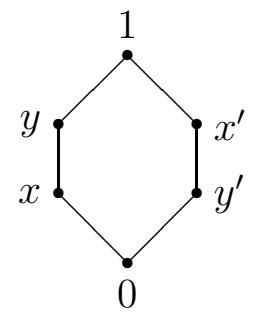

Figure 1: Ortholattice O6, also called benzene ring and hexagon.

On the one hand, the equations that hold in OML properly include those that hold in WOML, since WOML is a strictly more general class of lattices. But there is also a sense in which the equations of WOML can be considered to properly include those of OML, via a mapping that Theorem 2.11 below describes. First, we need a technical lemma.

Lemma 2.10 The following conditions hold in all WOMLs:

$$
a \equiv a=1
$$




$$
\begin{aligned}
& a \equiv b=1 \quad \Rightarrow \quad b \equiv a=1 \\
& a \equiv b=1 \quad \Rightarrow \quad a^{\prime} \equiv b^{\prime}=1 \\
& a \equiv b=1 \quad \Rightarrow \quad(a \cup c) \equiv(b \cup c)=1 \\
& a \equiv b=1 \quad \Rightarrow \quad(a \cap c) \equiv(b \cap c)=1 \\
& a \equiv b=1 \quad \& \quad b \equiv c=1 \quad \Rightarrow \quad a \equiv c=1 \\
& (a \cup b) \equiv(b \cup a)=1 \\
& ((a \cup b) \cup c) \equiv(a \cup(b \cup c))=1 \\
& a^{\prime \prime} \equiv a=1 \\
& \left(a \cup\left(b \cup b^{\prime}\right)\right) \equiv\left(b \cup b^{\prime}\right)=1 \\
& (a \cup(a \cap b)) \equiv a=1 \\
& (a \cap b) \equiv\left(a^{\prime} \cup b^{\prime}\right)^{\prime}=1 \\
& \left(a \cup\left(a^{\prime} \cap(a \cup b)\right)\right) \equiv(a \cup b)=1 \\
& a \equiv\left((a \cap b) \cup\left(a \cap b^{\prime}\right)\right)=a \equiv_{0}\left((a \cap b) \cup\left(a \cap b^{\prime}\right)\right) \\
& a=1 \quad \Leftrightarrow \quad a \equiv 1=1 \\
& a=1 \quad \Leftrightarrow \quad a \equiv_{0} 1=1
\end{aligned}
$$

In addition, Eqs. (14)-(16) and (20)-(29) hold in all ortholattices.

Proof. Most of these conditions are proved in [7], and the others are straightforward.

Theorem 2.11 The equational theory of OMLs can be simulated by a proper subset of the equational theory of WOMLs.

Proof. The equational theory of OML consists of equality axioms $(a=a$, $a=b \Rightarrow b=a, a=b \Rightarrow a^{\prime}=b^{\prime}, a=b \Rightarrow a \cup c=b \cup c, a=b \Rightarrow a \cap c=b \cap c$,

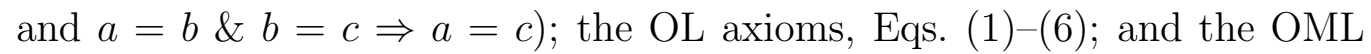
law, Eq. (12). Any theorem of the equational variety of OMLs can be proved with a sequence of applications of these axioms. We construct a mapping from these axioms into equations that hold in WOMLs as follows. We map each axiom, which is an equation in the form $t=s$ or an inference of the form $t_{1}=t_{2} \ldots \Rightarrow t=s$ (where $t, s$, and $t_{1}, t_{2}, \ldots$ are terms), to the equation $t \equiv s=1$ or the inference $t_{1} \equiv t_{2}=1 \ldots \Rightarrow t \equiv s=1$. These mappings hold in any WOML by Eqs. (14)-(26), respectively, of Lemma 2.10, We then simulate the OML proof by replacing each axiom reference in the proof with 
its corresponding WOML mapping. The result will be a proof that holds in the equational variety of WOMLs.

Such a mapped proof will use only a proper subset of the equations that hold in WOML: any equation whose right-hand side does not equal 1, such as $a=a$, will never be used.

Theorem 2.12 Let $t_{1}, \ldots, t_{n}, t$ be any terms $(n \geq 0)$. If the inference $t_{1}=$ $1 \& \ldots \& t_{n}=1 \Rightarrow t=1$ holds in all OMLs, then it holds in any WOML.

Proof. In any ortholattice, $t=1$ iff $t \equiv 1=1$ by Eq. (28). Therefore, the inference of the theorem can be restated as follows: $t_{1} \equiv 1=1 \& \ldots \& t_{n} \equiv$ $1=1 \Rightarrow t \equiv 1=1$. But this is exactly what we prove when we simulate the original OML proof of the inference in WOML, using the method in the proof of Theorem 2.11. Thus by Theorem 2.11, the inference holds in WOML.

Corollary 2.13 No set of equations of the form $t=1$ that hold in OML, when added to the equations of an ortholattice, determines the equational theory of OMLs.

Proof. Theorem 2.12 shows that all equations of this form hold in a WOML.

Lemma 2.14 In any WOML, $a C_{w} b$ iff $C(a, b)=1$.

Proof. In any OML, $a C b$ implies $a^{\prime} C b$. Therefore, by Theorem $2.11, a C_{w} b$ implies $a^{\prime} C_{w} b$ in any WOML. Using Eqs. (18) and (20) to combine these two conditions, we obtain $\left(a \cup a^{\prime}\right) \equiv\left(\left((a \cap b) \cup\left(a \cap b^{\prime}\right)\right) \cup\left(\left(a^{\prime} \cap b\right) \cup\left(a^{\prime} \cap b^{\prime}\right)\right)\right)=1$ i.e., $C(a, b) \equiv 1=1$, from which we obtain $C(a, b)=1$ by Eq. (28). Conversely, if $C(a, b)=1$, then in any OL, $1=(a \cap b) \cup\left(a \cap b^{\prime}\right) \cup\left(a^{\prime} \cap b\right) \cup\left(a^{\prime} \cap b^{\prime}\right) \leq$ $(a \cap b) \cup\left(a \cap b^{\prime}\right) \cup a^{\prime}=\left(a \cap\left((a \cap b) \cup\left(a \cap b^{\prime}\right)\right) \cup\left(a^{\prime} \cap\left((a \cap b) \cup\left(a \cap b^{\prime}\right)\right)^{\prime}\right)=\right.$ $a \equiv\left((a \cap b) \cup\left(a \cap b^{\prime}\right)\right)$, so $a C_{w} b$.

Theorem 2.15 (Foulis-Holland theorem, F-H) In any OML, if at least two of the three conditions $a C b, a C c$, and $b C c$ hold, then the distributive law $a \cap(b \cup c)=(a \cap b) \cup(a \cap c)$ holds.

Proof. See [12, p. 25]. 
Theorem 2.16 (Weak Foulis-Holland theorem, wF-H) In any WOML, if at least two of the three conditions $C(a, b)=1, C(a, c)=1$, and $C(b, c)=1$ hold, then the weak distributive law $(a \cap(b \cup c)) \equiv((a \cap b) \cup(a \cap c))=1$ holds.

Proof. By Lemma 2.14, we can replace the conditions with $a C_{w} b, a C_{w} c$, and $b C_{w} c$. Then the conclusion follows from $\mathrm{F}-\mathrm{H}$ and Theorem 2.11.

As Theorem 2.11 shows, if $t$ and $s$ are terms, then the equation $t \equiv s=1$ holds in all WOMLs iff the equation $t=s$ holds in all OMLs. One might naively expect, then, that if $t=s$ is the OML law, then $t \equiv s=1$ will be the WOML law. This is not always the case: the OML law given by Eq. (13), when converted to $\left(a \cup\left(a^{\prime} \cap(a \cup b)\right) \equiv(a \cup b)=1\right.$, is not the WOML law; in fact, it holds in any OL. However, there is a version of the OML law with this property, as the following theorem shows.

Theorem 2.17 An ortholattice is an OML iff it satisfies the following equation:

$$
a \cup\left(b \cap\left(a^{\prime} \cup b^{\prime}\right)\right)=a \cup b
$$

An ortholattice is a WOML iff it satisfies the following equation:

$$
\left(a \cup\left(b \cap\left(a^{\prime} \cup b^{\prime}\right)\right)\right) \equiv(a \cup b)=1
$$

Proof. For Eq. (30): It is easy to verify that Eq. (30) holds in an OML, for example by applying F-H: $a \cup\left(b \cap\left(a^{\prime} \cup b^{\prime}\right)\right)=(a \cup b) \cap\left(a \cup a^{\prime} \cup b\right)=$ $(a \cup b) \cap 1=a \cup b$. On the other hand, this equation fails in lattice O6 (Fig. (1), meaning it implies the orthomodular law by Theorem 2 of [12, p. 22]. It is also instructive to prove Eq. (13) directly: $a \cup\left(a^{\prime} \cap(a \cup b)\right)=a \cup\left((a \cup b) \cap a^{\prime}\right)=$ $a \cup\left((a \cup b) \cap\left(a^{\prime} \cup\left(a^{\prime} \cap b^{\prime}\right)\right)=a \cup\left((a \cup b) \cap\left(a^{\prime} \cup(a \cup b)^{\prime}\right)\right)=a \cup(a \cup b)=a \cup b\right.$, where the penultimate step follows from Eq. (30) with $a \cup b$ substituted for $b$, and all other steps hold in OL.

For Eq. (31): Since $a \cup\left(b \cap\left(a^{\prime} \cup b^{\prime}\right)\right)=a \cup b$ holds in any OML by Eq. (30), $\left(a^{\prime} \cup\left(b^{\prime} \cap(a \cup b)\right)\right) \equiv(a \cup b)=1$ holds in WOML by Theorem 2.11. On the other hand, substituting $b^{\prime}$ and $a^{\prime}$ for $a$ and $b$ in Eq. (31), we have $1=\left(b^{\prime} \cup\left(a^{\prime} \cap\left(b^{\prime \prime} \cup a^{\prime \prime}\right)\right)\right) \equiv\left(b^{\prime} \cup a^{\prime}\right)=\left(\left(b^{\prime} \cup\left(a^{\prime} \cap(b \cup a)\right)\right) \cap\left(b^{\prime} \cup a^{\prime}\right)\right) \cup((b \cap(a \cup$ $\left.\left.\left.\left(b^{\prime} \cap a^{\prime}\right)\right)\right) \cap(b \cap a)\right)=\left(b^{\prime} \cup\left(a^{\prime} \cap(b \cup a)\right) \cup(b \cap a)\right)=\left(a^{\prime} \cap(a \cup b)\right) \cup b^{\prime} \cup(a \cap b)$, which is the WOML law Eq. (9)).

Another version of the WOML law will be useful later. 
Theorem 2.18 An ortholattice is a WOML iff it satisfies the following condition:

$$
a \rightarrow_{1} b=1 \quad \Rightarrow \quad a \rightarrow_{2} b=1
$$

Proof. See Theorem 3.9 of [7].

\section{Distributive and Weakly Distributive Ortholattices}

Definition 3.1 (Pavičić and Megill [7]) An ortholattice in which the following equation holds:

$$
(a \equiv b) \cup\left(a \equiv b^{\prime}\right)=(a \cap b) \cup\left(a \cap b^{\prime}\right) \cup\left(a^{\prime} \cap b\right) \cup\left(a^{\prime} \cap b^{\prime}\right)=1
$$

is called a weakly distributive ortholattice, WDOL.

A WDOL is thus an ortholattice in which the condition $C(a, b)=1$ holds. This condition is known as commensurability. [9, Def. (2.13), p. 32].

Definition 3.2 An ortholattice to which the following condition is added:

$$
a \cap(b \cup c)=(a \cap b) \cup(a \cap c)
$$

is called a distributive ortholattice (DOL) or (much more often) a Boolean algebra (BA).

Eq. (34) is called the distributive law.

We recall that whereas every BA is a WDOL, there are WDOLs that are not BAs. [7] In particular, the lattice O6 (Fig. 1) is a WDOL but is not a BA.

The first part of the following theorem will turn out to be very useful, because it will let us reuse all of the results we have already obtained for WOMLs.

Theorem 3.3 Every WDOL is a WOML, but not every WOML is a WDOL. 


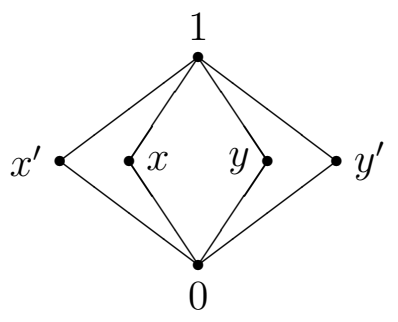

Figure 2: (a) OML M02;

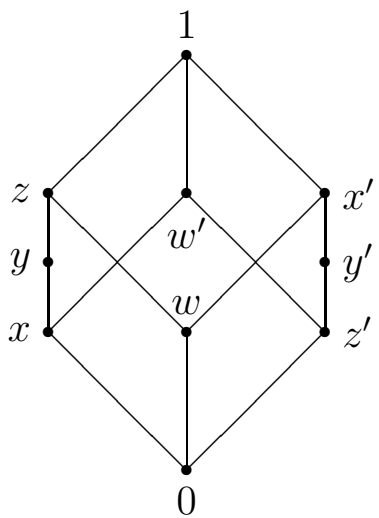

(b) Non-WDOL from [13], Fig. 3.

Proof. Since $a^{\prime} \cap b \leq a^{\prime} \cap(a \cup b)$ and $\left(a \cap b^{\prime}\right) \cup\left(a^{\prime} \cap b^{\prime}\right) \leq b^{\prime}$ in any OL, the WDOL law, Eq. (33), gives us $1=(a \cap b) \cup\left(a \cap b^{\prime}\right) \cup\left(a^{\prime} \cap b\right) \cup\left(a^{\prime} \cap b^{\prime}\right) \leq$ $\left(a^{\prime} \cap(a \cup b)\right) \cup b^{\prime} \cup(a \cap b)$, which is the WOML law, Eq. (9)).

On the other hand, the modular (and therefore WOML) lattice MO2 (Fig. 2a a) violates Eq. (33). If we put $x$ for $a$ and $y$ for $b$, the equation evaluates to $0=1$.

We are now in a position to prove two important equivalents to the WDOL law. We call them weak distributive laws, since they provide analogs to the distributive law of Boolean algebras.

Theorem 3.4 An ortholattice is a WDOL iff it satisfies either of the following equations:

$$
\begin{array}{r}
(a \cap(b \cup c)) \equiv_{0}((a \cap b) \cup(a \cap c))=1 \\
(a \cap(b \cup c)) \equiv((a \cap b) \cup(a \cap c))=1
\end{array}
$$

Proof. First, we prove these laws can be derived from each other in any OL. Assuming Eq. (35) and using the fact that $(a \cap b) \cup(a \cap c) \leq(a \cap(b \cup c)$, in any OL we have $1=\left((a \cap(b \cup c)) \rightarrow_{0}((a \cap b) \cup(a \cap c))\right) \cap\left(((a \cap b) \cup(a \cap c)) \rightarrow_{0}\right.$ $(a \cap(b \cup c)))=\left((a \cap(b \cup c)) \rightarrow_{0}((a \cap b) \cup(a \cap c))\right.$. Putting $b^{\prime}$ for $c$, $1=\left(\left(a \cap\left(b \cup b^{\prime}\right)\right) \rightarrow_{0}\left((a \cap b) \cup\left(a \cap b^{\prime}\right)\right)=\left(a \rightarrow_{0}\left((a \cap b) \cup\left(a \cap b^{\prime}\right)\right)=\right.\right.$ $\left(a^{\prime} \cup\left((a \cap b) \cup\left(a \cap b^{\prime}\right)\right) \leq\left(b^{\prime} \cap(b \cup a)\right) \cup a^{\prime} \cup(b \cap a)\right.$, which is the WOML law. This lets us use our previous WOML results.

Starting from the last equality in the first sentence of the previous paragraph, in any OL we also have $1=\left((a \cap(b \cup c)) \rightarrow_{0}((a \cap b) \cup(a \cap c))=(a \cap(b \cup c)) \rightarrow_{1}\right.$ 
$((a \cap b) \cup(a \cap c))=\left((a \cap(b \cup c)) \rightarrow_{1}((a \cap b) \cup(a \cap c))\right) \cap\left(((a \cap b) \cup(a \cap c)) \rightarrow_{1}\right.$ $(a \cap(b \cup c)))$. Therefore, using the footnote to Definition 2.3 and Theorem 2.12, it follows that in any WOML, and therefore (by the previous paragraph) in any OL, Eq. (35) implies $1=(a \cap(b \cup c)) \equiv((a \cap b) \cup(a \cap c))$.

Conversely, Eq. (35) follows immediately from Eq. (36) in any OL. Thus these two equations are equivalent laws when added to the equations for OL. Next, we prove that Eq. (36) is equivalent to the WDOL law in the presence of the equations for OL.

Since $C(a, b)=1$ for any $a, b$ in a WDOL, Eq. (36) follows immediately from wF-H (Theorem 2.16), which holds in every WOML and thus, by Theorem 3.3, in every WDOL.

Conversely, in OML, we can prove $C(a, b)=1$ if we use instances of the distributive law as hypotheses. Using Theorem 2.11, such a proof can be converted to a WOML proof, replacing the instances of the distributive law with instances of Eq. (36). This will yield a proof of $C(a, b) \equiv 1=1$, which in any OL implies $C(a, b)=1$ by Eq. (28). This proves that Eq. (36) implies the WDOL law, Eq. (33).

Theorem 3.5 An ortholattice is a WDOL iff it satisfies either of the following equations:

$$
\begin{gathered}
a \equiv\left((a \cap b) \cup\left(a \cap b^{\prime}\right)\right)=1 \\
a \equiv_{0}\left((a \cap b) \cup\left(a \cap b^{\prime}\right)\right)=1 .
\end{gathered}
$$

Proof. In any OL, $a \equiv\left((a \cap b) \cup\left(a \cap b^{\prime}\right)\right)=\left(a \cap\left((a \cap b) \cup\left(a \cap b^{\prime}\right)\right)\right) \cup\left(a^{\prime} \cap\right.$ $\left.\left(\left(a^{\prime} \cup b^{\prime}\right) \cap\left(a^{\prime} \cup b\right)\right)\right)=\left((a \cap b) \cup\left(a \cap b^{\prime}\right)\right) \cup a^{\prime}=\left(a \rightarrow_{0} b\right)^{\prime} \cup\left(a \rightarrow_{1} b\right)$. Thus Eq. (37) implies $1=\left(a \rightarrow_{0} b\right)^{\prime} \cup\left(a \rightarrow_{1} b\right) \leq\left(a \rightarrow_{2} b\right)^{\prime} \cup\left(a \rightarrow_{1} b\right)$, which is the WOML law in the form of Eq. (10). By Lemma 2.14, in any WOML Eq. (37) implies $C(a, b)=1$, which is the WDOL law.

For the converse, Eq. (37) holds in an WDOL by Lemma 2.14.

Eq. (38) is equivalent to Eq. (37) in any OL by Eq. (27).

We mention that Eq. (37) is the definition of $a C_{w} b$.

Theorem 3.6 An ortholattice is a WDOL iff it satisfies the following condition:

$$
a \equiv_{0} b=1 \quad \Rightarrow \quad(a \cup c) \equiv_{0}(b \cup c)=1
$$


Proof. First, we show that Eq. (39) implies the WOML law. Putting $d$ for $a$ and $d \cap e$ for $b$, the hypothesis becomes, in an OL, $1=d \equiv_{0}(d \cap e)=$ $\left(d^{\prime} \cup(d \cap e)\right) \cap\left(\left(d^{\prime} \cup e^{\prime}\right) \cup d\right)=\left(d^{\prime} \cup(d \cap e)\right) \cap 1=d \rightarrow_{1} e$. Also putting $e$ for $c$, the conclusion becomes, in an OL, $1=(d \cup e) \equiv_{0}((d \cap e) \cup e)=(d \cup e) \equiv_{0}$ $e=\left(\left(d^{\prime} \cap e^{\prime}\right) \cup e\right) \cap\left(e^{\prime} \cup(d \cup e)\right)=\left(\left(d^{\prime} \cap e^{\prime}\right) \cup e\right) \cap 1=d \rightarrow_{2} e$. The condition $d \rightarrow_{1} e=1 \Rightarrow d \rightarrow_{2} e=1$ is the WOML law by Eq. (32).

Having our previous WOML results now available to us, we next show that Eq. (39) implies the WDOL law. We put $d^{\prime} \cap\left(d \cup e^{\prime}\right)$ for $a, e^{\prime} \cap\left(e \cup d^{\prime}\right)$ for $b$, and $d^{\prime}$ for $c$. To satisfy the hypothesis, we must show that in any WOML, $\left(d^{\prime} \cap\left(d \cup e^{\prime}\right)\right) \equiv_{0}\left(e^{\prime} \cap\left(e \cup d^{\prime}\right)\right)=1$, i.e., that $\left(\left(d \cup\left(d^{\prime} \cap e\right)\right) \cup\left(e^{\prime} \cap\left(e \cup d^{\prime}\right)\right)\right) \cap$ $\left(\left(e \cup\left(e^{\prime} \cap d\right)\right) \cup\left(d^{\prime} \cap\left(d \cup e^{\prime}\right)\right)\right)=1$. For the first conjunct, we apply $\mathrm{wF}-\mathrm{H}$ to $\left(d \cup\left(d^{\prime} \cap e\right)\right) \cup\left(e^{\prime} \cap\left(e \cup d^{\prime}\right)\right)=\left(\left(d \cup\left(d^{\prime} \cap e\right)\right) \cup\left(e^{\prime} \cap\left(e \cup d^{\prime}\right)\right)\right) \equiv 1=1$ to obtain $\left.)\left(d \cup\left(d^{\prime} \cap e\right) \cup e^{\prime}\right) \cap\left(d \cup\left(d^{\prime} \cap e\right) \cup e \cup d^{\prime}\right)\right) \equiv 1=1$, which reduces to $(1 \cap 1) \equiv 1=1$. The other conjunct is satisfied similarly, by symmetry. The conclusion becomes $\left(\left(d^{\prime} \cap\left(d \cup e^{\prime}\right)\right) \cup d^{\prime}\right) \equiv_{0}\left(\left(e^{\prime} \cap\left(e \cup d^{\prime}\right)\right) \cup d^{\prime}\right)=d^{\prime} \equiv_{0}$ $\left(\left(e^{\prime} \cap\left(e \cup d^{\prime}\right)\right) \cup d^{\prime}\right)=1$. Expanding the definition of $\equiv_{0}$ and discarding the left-hand conjunct, we have $\left(\left(e \cup\left(e^{\prime} \cap d\right)\right) \cap d\right) \cup d^{\prime}=1$. Using wF-H, this becomes $1=\left((e \cap d) \cup\left(\left(e^{\prime} \cap d\right) \cap d\right)\right) \cup d^{\prime}=\left((e \cap d) \cup\left(e^{\prime} \cap d\right)\right) \cup d^{\prime}=$ $\left(\left((e \cap d) \cup\left(e^{\prime} \cap d\right)\right) \cup d^{\prime}\right) \equiv 1$. Conjoining both sides of the $\equiv$ with $d$ using Eq. (18), we have $\left(\left(\left((e \cap d) \cup\left(e^{\prime} \cap d\right)\right) \cup d^{\prime}\right) \cap d\right) \equiv(1 \cap d)=1$. Applying wF-H twice, we obtain $1=\left(\left(((e \cap d) \cap d) \cup\left(\left(e^{\prime} \cap d\right) \cap d\right)\right) \cup\left(d^{\prime} \cap d\right)\right) \equiv(1 \cap d)=$ $\left(\left((e \cap d) \cup\left(e^{\prime} \cap d\right)\right) \cup 0\right) \equiv d=\left((e \cap d) \cup\left(e^{\prime} \cap d\right)\right) \equiv d$, which is the WDOL law in the form of Eq. (37).

Conversely, to show that Eq. (39) holds in any WDOL, we apply Eq. (40) below (which does not depend on the present theorem) to the hypothesis and conclusion, converting it to Eq. (17).

An essential characteristic of the WDOL law and its equivalents is that they must fail in the modular (and therefore OML and WOML) lattice MO2. However, such a failure is not sufficient to ensure that we have a WDOL law equivalent.

Theorem 3.7 The following condition holds in all WDOLs:

$$
a \equiv_{0} b=1 \quad \Leftrightarrow \quad a \equiv b=1
$$

It also fails in modular lattice MO2. However, when added to the equations for OL, it does not determine the equations of WDOL.

Proof. To verify that this condition holds in any WDOL, we first convert the hypothesis to the OL-equivalent hypothesis $\left(a \equiv_{0} b\right) \equiv 1=1$ using Eq. (29)). 
By using the WDOL law $C(a, b)=1$ to satisfy the hypotheses of any uses of $\mathrm{wF}-\mathrm{H}$, it is then easy to prove that this condition holds in any WDOL. In particular, the reverse implication holds in any OL.

The failure of Eq. (40) in MO2 is verified by putting $x$ for $a$ and $y$ for $b$; then the left-hand side holds but the right-hand side becomes $0=1$. On the other hand, it does not imply the WDOL law nor even the WOML law: it passes in the non-WOML lattice of Figure $2 \mathrm{~b}$.

On the one hand, the equations that hold in BA properly include those that hold in WDOL, since WDOL is a strictly more general class of lattices. But there is also a sense in which the equations of WDOL can be considered to properly include those of BA, via a mapping that Theorem 3.8 below describes.

Theorem 3.8 The equational theory of BAs can be simulated by a proper subset of the equational theory of WDOLs.

Proof. The equational theory of BA consists of equality axioms (see the proof of Theorem 2.11); the OL axioms, Eqs. (1)-(6); and the distributive law, Eq. (34). Any theorem of the equational variety of BAs can be proved with a sequence of applications of these axioms. We construct a mapping from these axioms into equations that hold in WDOLs as follows. We map each axiom, which is an equation in the form $t=s$ or an inference of the form $t_{1}=t_{2} \ldots \Rightarrow t=s$ (where $t, s$, and $t_{1}, t_{2}, \ldots$ are terms), to the equation $t \equiv_{0} s=1$ or the inference $t_{1} \equiv_{0} t_{2}=1 \ldots \Rightarrow t \equiv_{0} s=1$. These mappings hold in any WDOL by Eqs. (14)-(25) and (35), respectively, after converting $\equiv$ to $\equiv_{0}$ with Eq. (40). We then simulate the BA proof by replacing each axiom reference in the proof with its corresponding WDOL mapping. The result will be a proof that holds in the equational variety of WDOLs.

Such a mapped proof will use only a proper subset of the equations that hold in WDOL: any equation whose right-hand side does not equal 1, such as $a=a$, will never be used.

Theorem 3.9 Let $t_{1}, \ldots, t_{n}, t$ be any terms $(n \geq 0)$. If the inference $t_{1}=$ $1 \& \ldots \& t_{n}=1 \Rightarrow t=1$ holds in all BAs, then it holds in any WDOL.

Proof. In any ortholattice, $t=1$ iff $t \equiv_{0} 1=1$ by Eq. (29). Therefore, the inference of the theorem can be restated as follows: $t_{1} \equiv_{0} 1=1 \& \ldots \& t_{n} \equiv_{0}$ $1=1 \Rightarrow t \equiv_{0} 1=1$. But this is exactly what we prove when we simulate 
the original BA proof of the inference in WDOL, using the method in the proof of Theorem 3.8. Thus by Theorem 3.8, the inference holds in WDOL.

Corollary 3.10 No set of equations of the form $t=1$ that hold in BA, when added to the equations of an ortholattice, determines the equational theory of $\mathrm{BA} s$.

Proof. Theorem 3.9 shows that all equations of this form hold in a WDOL.

\section{The Classes of Proper Weakly Orthomodular and Proper Weakly Distributive Ortholattices}

One of the main aims of our paper is to prove that both quantum and classical logics are sound and complete with respect to at least a class of all weakly orthomodular lattices (WOMLs) in which orthomodularity fails for every lattice and a class of all weakly distributive lattices (WDOLs) in which distributivity fails for every lattice, respectively.

To prove the soundness and completeness of quantum logic we shall consider a new class of lattices that belong to the class WOML but not to the class OML. We will denote the resulting class WOML-OML. In other words, WOML-OML denotes the set-theoretical difference WOML\OML. A member of the class WOML-OML is a lattice, specifically a member of the class WOML, and we will call such a lattice a proper WOML. Thus a proper WOML is one that satisfies the WOML equations but violates the OML equations. Lattice O6 is an example of a proper WOML. Lattice MO2 is an example of a WOML that is not a proper WOML, i.e., that does not belong to the class WOML-OML, since it also belongs to the class OML.

Notice that WOML-OML is not an equational variety like WOML, because we cannot turn WOML into WOML-OML by adding new equational conditions to those defining WOML. If we try to add the orthomodularity condition (12) [14, 11] to WOML-OML, we will get the empty set.

In Section 6 we shall show that quantum logics is complete for WOMLOML: every wff whose valuation equals 1 for all members of WOML-OML 
is a provable statement in quantum logic. This is not necessarily obvious a priori: quantum logic $(\mathcal{Q} \mathcal{L})$ is not necessarily complete for an arbitrary collection of WOMLs. For example, it is not complete for the subset of WOML-OML consisting of the singleton set $\{\mathrm{O} 6\}$, since O6 is a model for classical logic.

The significance of this result can be explained as follows. Since $\mathcal{Q L}$ is already complete for OML models, it might be argued that completeness for the more general WOML models ([7]) has its origin in the OML members of the equational variety WOML, rather than being an intrinsic property of the non-OML members. We show that this is not the case by completely removing all OMLs from the picture.

In order for the completeness proof to go through, we will have to construct a special Lindenbaum algebra that belongs to WOML-OML. This requires a modification to the standard Lindenbaum algebra (which, in the standard proof, "wants" to be an OML). The technique that we use, involving cutting down the equivalence classes for the Lindenbaum algebra to force it to belong to WOML-OML, might be useful for other completeness proofs that are not amenable to the standard Lindenbaum-algebra approach.

Following an analogous blueprint, in Section 7 we will also show that classical logic is complete for the class of models WDOL-BA, defined as the set-theoretical difference WDOL $\backslash$ BA (where WDOL and BA here denote equational varieties), which again by definition has nothing to do with Boolean algebras. In fact, a simpler result is possible: Schechter [15, p. 272] has proved that classical logic $(\mathcal{C L})$ is complete for the single WDOL lattice O6. Schechter's result can be strengthened to show that classical logic is complete for any subset of WDOL. This is an immediate consequence of the fact that classical logic is maximal, i.e., no extension of it can be consistent. So if classical logic is sound for a model, it is automatically complete for that model.

\section{$5 \quad$ Logics and Their Soundness for Our Models}

Logic $(\mathcal{L})$ is a language consisting of propositions and a set of conditions and rules imposed on them called axioms and rules of inference.

The propositions we use are well-formed formulae (wffs), defined as fol- 
lows. We denote elementary, or primitive, propositions by $p_{0}, p_{1}, p_{2}, \ldots$, and have the following primitive connectives: $\neg$ (negation) and $\vee$ (disjunction). The set of wffs is defined recursively as follows:

$p_{j}$ is a wff for $j=0,1,2, \ldots$

$\neg A$ is a wff if $A$ is a wff.

$A \vee B$ is a wff if $A$ and $B$ are wffs.

We introduce conjunction with the following definition:

Definition $5.1 A \wedge B \stackrel{\text { def }}{=} \neg(\neg A \vee \neg B)$.

The operations of implication are the following ones (classical, Sasaki, and Kalmbach) [16]:

Definition 5.2 $A \rightarrow_{0} B \stackrel{\text { def }}{=} \neg A \vee B$.

Definition 5.3 $A \rightarrow_{1} B \stackrel{\text { def }}{=} \neg A \vee(A \wedge B)$

Definition 5.4 $\quad A \rightarrow_{3} B \stackrel{\text { def }}{=}(\neg A \wedge B) \vee(\neg A \wedge \neg B) \vee(A \wedge(\neg A \vee B))$.

We also define the equivalence operations as follows:

Definition 5.5 $\quad A \equiv B \stackrel{\text { def }}{=}(A \wedge B) \vee(\neg A \wedge \neg B)$.

Definition 5.6 $A \equiv_{0} B \stackrel{\text { def }}{=}\left(A \rightarrow_{0} B\right) \wedge\left(B \rightarrow_{0} A\right)$.

Connectives bind from weakest to strongest in the order $\rightarrow, \equiv, \vee, \wedge, \neg$.

Let $\mathcal{F}^{\circ}$ be the set of all propositions, i.e., of all wffs. Of the above connectives, $\vee$ and $\neg$ are primitive ones. Wffs containing $\vee$ and $\neg$ within $\operatorname{logic} \mathcal{L}$ are used to build an algebra $\mathcal{F}=\left\langle\mathcal{F}^{\circ}, \neg, \vee\right\rangle$. In $\mathcal{L}$, a set of axioms and rules of inference are imposed on $\mathcal{F}$. From a set of axioms by means of rules of inference, we get other expressions which we call theorems. Axioms themselves are also theorems. A special symbol $\vdash$ is used to denote the set of theorems. Hence $A \in \vdash$ iff $A$ is a theorem. The statement $A \in \vdash$ is usually written as $\vdash A$. We read this: " $A$ is provable" since if $A$ is a theorem, then there is a proof for it. We present the axiom systems of our propositional logics in schemata form (so that we dispense with the rule of substitution). 


\subsection{Quantum Logic and Its Soundness for WOML-OML Models}

We present Kalmbach's quantum logic because it is the system that has been investigated in the greatest detail in her book [12] and elsewhere [17, 13]. Quantum logic $(\mathcal{Q L})$ is defined as a language consisting of propositions and connectives (operations) as introduced above, and the following axioms and a rule of inference. We will use $\vdash_{\mathcal{Q L}}$ to denote provability from the axioms and rule of $\mathcal{Q} \mathcal{L}$ and omit the subscript when it is clear from context (such as in the list of axioms that follow).

\section{Axioms}

$\begin{array}{ll}\text { A1 } & \vdash A \equiv A \\ \text { A2 } & \vdash A \equiv B \rightarrow_{0}\left(B \equiv C \rightarrow_{0} A \equiv C\right) \\ \text { A3 } & \vdash A \equiv B \rightarrow_{0} \neg A \equiv \neg B \\ \text { A4 } & \vdash A \equiv B \rightarrow_{0} A \wedge C \equiv B \wedge C \\ \text { A5 } & \vdash A \wedge B \equiv B \wedge A \\ \text { A6 } & \vdash A \wedge(B \wedge C) \equiv(A \wedge B) \wedge C \\ \text { A7 } & \vdash A \wedge(A \vee B) \equiv A \\ \text { A8 } & \vdash \neg A \wedge A \equiv(\neg A \wedge A) \wedge B \\ \text { A9 } & \vdash A \equiv \neg \neg A \\ \text { A10 } & \vdash \neg(A \vee B) \equiv \neg A \wedge \neg B \\ \text { A11 } & \vdash A \vee(\neg A \wedge(A \vee B)) \equiv A \vee B \\ \text { A12 } & \vdash(A \equiv B) \equiv(B \equiv A) \\ \text { A13 } & \vdash A \equiv B \rightarrow_{0}\left(A \rightarrow \rightarrow_{0} B\right) \\ \text { A14 } & \vdash\left(A \rightarrow \rightarrow_{0} B\right) \rightarrow_{3}\left(A \rightarrow_{3}\left(A \rightarrow_{3} B\right)\right) \\ \text { A15 } & \vdash\left(A \rightarrow \rightarrow_{3} B\right) \rightarrow_{0}\left(A \rightarrow_{0} B\right)\end{array}$

Rule of Inference (Modus Ponens)

$$
\mathrm{R} 1 \quad \vdash A \quad \& \quad \vdash A \rightarrow_{3} B \Rightarrow \vdash B
$$

In Kalmbach's presentation, the connectives $\vee, \wedge$, and $\neg$ are primitive. In the base set of any model (such as an OML or WOML model) that belongs to OL, $\cap$ can be defined in terms of $\cup$ and ', as justified by DeMorgan's law, and thus the corresponding $\wedge$ can be defined in terms of $\vee$ and $\neg$ [using 
Eq. (6)]. We shall do this for simplicity. Regardless of whether we consider $\wedge$ primitive or defined, we can drop axioms A1, A11, and A15 because it has been proved that they are redundant, i.e., can be derived from the other axioms. [13]

Definition 5.7 For $\Gamma \subseteq \mathcal{F}^{\circ}$ we say $A$ is derivable from $\Gamma$ and write $\Gamma \vdash_{\mathcal{Q L}} A$ or just $\Gamma \vdash A$ if there is a finite sequence of formulae, the last of which is $A$, and each of which is either one of the axioms of $\mathcal{Q L}$ or is a member of $\Gamma$ or is obtained from its precursors with the help of a rule of inference of the logic.

To prove soundness means to prove that all axioms as well as the rules of inference (and therefore all theorems) of $\mathcal{Q} \mathcal{L}$ hold in its models.

Definition 5.8 We call $\mathcal{M}=\langle L, h\rangle$ a model if $L$ is an algebra and $h$ : $\mathcal{F}^{\circ} \longrightarrow L$, called a valuation, is a morphism of formulae $\mathcal{F}^{\circ}$ into L, preserving the operations $\neg, \vee$ while turning them into ${ }^{\prime}, \cup$.

Whenever the base set $L$ of a model belongs to WOML-OML, we say (informally) that the model belongs to WOML-OML. In particular, if we say "for all models in WOML-OML" or "for all proper WOML models," we mean for all base sets in WOML-OML and for all valuations on each base set. The term "model" may refer either to a specific pair $\langle L, h\rangle$ or to all possible such pairs with the base set $L$, depending on context.

Definition 5.9 We call a formula $A \in \mathcal{F}^{\circ}$ valid in the model $\mathcal{M}$, and write $\vDash_{\mathcal{M}} A$, if $h(A)=1$ for all valuations $h$ on the model, i.e., for all $h$ associated with the base set $L$ of the model. We call a formula $A \in \mathcal{F}^{\circ}$ a consequence of $\Gamma \subseteq \mathcal{F}^{\circ}$ in the model $\mathcal{M}$ and write $\Gamma \vDash_{\mathcal{M}} A$ if $h(X)=1$ for all $X$ in $\Gamma$ implies $h(A)=1$, for all valuations $h$.

For brevity, whenever we do not make it explicit, the notations $\vDash_{\mathcal{M}} A$ and $\Gamma \vDash_{\mathcal{M}} A$ will always be implicitly quantified over all models of the appropriate type, in this section for all proper WOML models $\mathcal{M}$. Similarly, when we say "valid" without qualification, we will mean valid in all models of that type.

The following theorem shows that if $A$ is a theorem of $\mathcal{Q L}$, then $A$ is valid in any proper WOML model. 
In [7, 8] we proved the soundness for WOML and OML. We now prove the soundness of quantum logic by means of WOML-OML, i.e., that if $A$ is a theorem in $\mathcal{Q L}$, then $A$ is valid in any proper WOML model, i.e., in any WOML-OML model.

Theorem 5.10 [Soundness] $\quad \Gamma \vdash A \Rightarrow \Gamma \vDash_{\mathcal{M}} A$

Proof. By Theorem 29 of [18], any WOML is a model for $\mathcal{Q L}$. Therefore, any proper WOML is also a model.

\subsection{Classical Logic and Its Soundness for WDOL-BA Models}

We make use of the PM classical logical system $\mathcal{C L}$ (Whitehead and Russell's Principia Mathematica axiomatization in Hilbert and Ackermann's presentation [19] but in schemata form so that we dispense with their rule of substitution). In this system, the connectives $\vee$ and $\neg$ are primitive, and the $\rightarrow_{0}$ connective shown in the axioms is implicitly understood to be expanded according to its definition. We will use $\vdash_{\mathcal{C L}}$ to denote provability from the axioms and rule of $\mathcal{C} \mathcal{L}$, omitting the subscript when it is clear from context.

Axioms

$$
\begin{array}{ll}
\mathrm{A} 1 & \vdash A \vee A \rightarrow_{0} A \\
\mathrm{~A} 2 & \vdash A \rightarrow_{0} A \vee B \\
\mathrm{~A} 3 & \vdash A \vee B \rightarrow_{0} B \vee A \\
\mathrm{~A} 4 & \vdash\left(A \rightarrow_{0} B\right) \rightarrow_{0}\left(C \vee A \rightarrow_{0} C \vee B\right)
\end{array}
$$

Rule of Inference (Modus Ponens)

$$
\mathrm{R} 1 \quad \vdash A \quad \& \quad A \rightarrow_{0} B \quad \Rightarrow \quad \vdash B
$$

We assume that the only legitimate way of inferring theorems in $\mathcal{C} \mathcal{L}$ is by means of these axioms and the Modus Ponens rule. We make no assumption about valuations of the primitive propositions from which wffs are built, but instead are interested in wffs that are valid in the underlying models. Soundness and completeness will show that those theorems that can be inferred from the axioms and the rule of inference are exactly those that are valid. 
We define derivability in $\mathcal{C} \mathcal{L}, \Gamma \vdash_{\mathcal{C L}} A$ or just $\Gamma \vdash A$, in the same way as we do for system $\mathcal{Q L}$. The models and validity of formulae in a model are also defined as for $\mathcal{Q L}$ above.

The following theorem shows that if $A$ is a theorem of $\mathcal{C} \mathcal{L}$, then $A$ is valid in any proper WDOL model.

In [7, 8] we proved the soundness for WDOL and BA. We now prove the soundness of classical logic by means of WDOL-BA, i.e., that if $A$ is a theorem in $\mathcal{C L}$, then $A$ is valid in any proper WDOL model, i.e., in any WDOL-BA model.

Theorem 5.11 [Soundness] $\quad \Gamma \vdash A \Rightarrow \Gamma \vDash_{\mathcal{M}} A$

Proof. By Theorem 30 of [18, any WDOL is a model for $\mathcal{C L}$. Therefore, any proper WDOL is also a model.

\section{The Completeness of Quantum Logic for WOML-OML Models}

Our main task in proving the soundness of $\mathcal{Q} \mathcal{L}$ in the previous section was to show that all axioms as well as the rules of inference (and therefore all theorems) from $\mathcal{Q L}$ hold in WOML-OML. The task of proving the completeness of $\mathcal{Q} \mathcal{L}$ is the opposite one: we have to impose the structure of WOML-OML on the set $\mathcal{F}^{\circ}$ of formulae of $\mathcal{Q L}$.

We start with a relation of congruence, i.e., a relation of equivalence compatible with the operations in $\mathcal{Q} \mathcal{L}$. We make use of an equivalence relation to establish a correspondence between formulae of $\mathcal{Q L}$ and formulae of WOML-OML. The resulting equivalence classes stand for elements of a proper WOML (i.e., a member of WOML-OML) and enable the completeness proof of $\mathcal{Q L}$ by means of WOML-OML.

Our definition of congruence involves a special set of valuations on lattice O6 (shown in Figure 1i) called $\mathcal{O} 6$ and defined as follows.

Definition 6.1 Letting O6 represent the lattice from Figure 1, we define $\mathcal{O} 6$ as the set of all mappings o: $\mathcal{F}^{\circ} \longrightarrow$ O6 such that for $A, B \in \mathcal{F}^{\circ}$, $o(\neg A)=o(A)^{\prime}$, and $o(A \vee B)=o(A) \cup o(B)$.

The purpose of $\mathcal{O} 6$ is to let us refine the equivalence classes used for the completeness proof, so that the Lindenbaum algebra will be a proper WOML, 
i.e., one that is not orthomodular. This is accomplished by conjoining the term $(\forall o \in \mathcal{O} 6)[(\forall X \in \Gamma)(o(X)=1) \Rightarrow o(A)=o(B)]$ to the equivalence relation definition, meaning that for equivalence we require also that (whenever the valuations $o$ of the wffs in $\Gamma$ are all 1) the valuations of wffs $A$ and $B$ map to the same point in the lattice O6. Thus wffs $A \vee B$ and $A \vee(\neg A \wedge(A \vee B))$ become members of two separate equivalence classes, what by Theorem 6.7 below, amounts to non-orthomodularity of WOML. Without the conjoined term, these two wffs would belong to the same equivalence class. The point of doing this is to provide a completeness proof that is not dependent in any way on the orthomodular law and to show that completeness does not require that any of the underlying models be OMLs.

Theorem 6.2 The relation of equivalence $\approx_{\Gamma, \mathcal{Q L}}$ or just $\approx$, defined as

$$
\begin{aligned}
& A \approx B \\
& \stackrel{\text { def }}{=} \Gamma \vdash A \equiv B \&(\forall o \in \mathcal{O} 6)[(\forall X \in \Gamma)(o(X)=1) \Rightarrow o(A)=o(B)],
\end{aligned}
$$

is a relation of congruence in the algebra $\mathcal{F}$, where $\Gamma \subseteq \mathcal{F}^{\circ}$

Proof. Let us first prove that $\approx$ is an equivalence relation. $A \approx A$ follows from A1 [Eq. (41)] of system $\mathcal{Q} \mathcal{L}$ and the identity law of equality. If $\Gamma \vdash A \equiv B$, we can detach the left-hand side of A12 to conclude $\Gamma \vdash B \equiv A$, through the use of A13 and repeated uses of A14 and R1. From this and commutativity of equality, we conclude $A \approx B \Rightarrow B \approx A$. (For brevity we will not usually mention further uses of A12, A13, A14, and R1 in what follows.) The proof of transitivity runs as follows.

$$
\begin{gathered}
A \approx B \quad \& \quad B \approx C \\
\Rightarrow \Gamma \vdash A \equiv B \quad \& \quad \Gamma \vdash B \equiv C \\
\&(\forall o \in \mathcal{O} 6)[(\forall X \in \Gamma)(o(X)=1) \Rightarrow o(A)=o(B)] \\
\&(\forall o \in \mathcal{O} 6)[(\forall X \in \Gamma)(o(X)=1) \Rightarrow o(B)=o(C)] \\
\Rightarrow \Gamma \vdash A \equiv C \\
\&(\forall o \in \mathcal{O} 6)[(\forall X \in \Gamma)(o(X)=1) \Rightarrow o(A)=o(B) \& o(B)=o(C)] .
\end{gathered}
$$

In the last line above, $\Gamma \vdash A \equiv C$ follows from A2, and the last metaconjunction reduces to $o(A)=o(C)$ by transitivity of equality. Hence the conclusion $A \approx C$ by definition. 
In order to be a relation of congruence, the relation of equivalence must be compatible with the operations $\neg$ and $\vee$. These proofs run as follows.

$A \approx B$

$$
\begin{aligned}
& \Rightarrow \Gamma \vdash A \equiv B \\
& \& \quad(\forall o \in \mathcal{O} 6)[(\forall X \in \Gamma)(o(X)=1) \Rightarrow o(A)=o(B)] \\
& \quad \Rightarrow \Gamma \vdash \neg A \equiv \neg B \\
& \& \quad(\forall o \in \mathcal{O} 6)\left[(\forall X \in \Gamma)(o(X)=1) \Rightarrow o(A)^{\prime}=o(B)^{\prime}\right] \\
& \quad \Rightarrow \Gamma \vdash \neg A \equiv \neg B \\
& \& \quad(\forall o \in \mathcal{O} 6)[(\forall X \in \Gamma)(o(X)=1) \Rightarrow o(\neg A)=o(\neg B)] \\
& \quad \Rightarrow \neg A \approx \neg B
\end{aligned}
$$

$A \approx B$

$$
\begin{aligned}
\Rightarrow & \Gamma \vdash A \equiv B \\
\& \quad & (\forall o \in \mathcal{O} 6)[(\forall X \in \Gamma)(o(X)=1) \Rightarrow o(A)=o(B)] \\
& \Rightarrow \Gamma \vdash(A \vee C) \equiv(B \vee C) \\
\& \quad(\forall o & \in \mathcal{O} 6)[(\forall X \in \Gamma)(o(X)=1) \Rightarrow o(A) \cup o(C)=o(B) \cup o(C)] \\
\Rightarrow(A \vee C) \approx(B \vee C) &
\end{aligned}
$$

In the second step of Eq. (64), we used A3. In the second step of Eq. (65), we used A4 and A10. For the quantified part of these expressions, we applied the definition of $\mathcal{O} 6$.

Definition 6.3 The equivalence class for wff $A$ under the relation of equivalence $\approx$ is defined as $|A|=\left\{B \in \mathcal{F}^{\circ}: A \approx B\right\}$, and we denote $\mathcal{F}^{\circ} / \approx$ $=\left\{|A|: A \in \mathcal{F}^{\circ}\right\}$. The equivalence classes define the natural morphism $f: \mathcal{F}^{\circ} \longrightarrow \mathcal{F}^{\circ} / \approx$, which gives $f(A)={ }^{\text {def }}|A|$. We write $a=f(A)$, $b=f(B)$, etc.

Lemma 6.4 The relation $a=b$ on $\mathcal{F}^{\circ} / \approx$ is given by:

$$
|A|=|B| \quad \Leftrightarrow \quad A \approx B
$$

Lemma 6.5 The Lindenbaum algebra $\mathcal{A}=\left\langle\mathcal{F}^{\circ} / \approx, \neg / \approx, \vee / \approx\right\rangle$ is a WOML, i.e., Eqs. (1)-(6) and Eq. (9) hold for $\neg / \approx$ and $\vee / \approx$ as' and $\cup$ respectively [where - for simplicity - we use the same symbols (' and $\cup$ ) as for O6, since there are no ambiguous expressions in which the origin of the operations would not be clear from the context]. 
Proof. For the $\Gamma \vdash A \equiv B$ part of the $A \approx B$ definition, the proofs of the ortholattice conditions, Eqs. (11)-(6), follow from A5, A6, A9, the dual of A8, the dual of A7, and DeMorgan's laws respectively. (The duals follow from DeMorgan's laws, derived from A10, A9, and A3.) A11 gives us an analog of the OML law for the $\Gamma \vdash A \equiv B$ part, and the WOML law Eq. (9) follows from the OML law in an ortholattice. For the quantified part of the $A \approx B$ definition, lattice $\mathrm{O} 6$ is a (proper) WOML.

Lemma 6.6 In the Lindenbaum algebra $\mathcal{A}$, if $f(X)=1$ for all $X$ in $\Gamma$ implies $f(A)=1$, then $\Gamma \vdash A$.

Proof. Let us assume that $f(X)=1$ for all $X$ in $\Gamma$ implies $f(A)=1$ i.e., $|A|=1=|A| \cup|A|^{\prime}=|A \vee \neg A|$, where the first equality is from Definition 6.3, the second equality follows from Eq. (7) (the definition of 1 in an ortholattice), and the third from the fact that $\approx$ is a congruence. Thus $A \approx(A \vee \neg A)$, which by definition means $\Gamma \vdash A \equiv(A \vee \neg A) \&(\forall o \in \mathcal{O} 6)[(\forall X \in \Gamma)(o(X)=1) \Rightarrow$ $o(A)=o((A \vee \neg A))]$. This implies, in particular (by dropping the second conjunct), $\Gamma \vdash A \equiv(A \vee \neg A)$. Now in any ortholattice, $a \equiv\left(a \cup a^{\prime}\right)=a$ holds. By mapping the steps in the proof of this ortholattice identity to steps in a proof in the logic, we can prove $\vdash(A \equiv(A \vee \neg A)) \equiv A$ from $\mathcal{Q} \mathcal{L}$ axioms A1-A15. (We call this a "proof by analogy," which is closely related to the method of Theorem 2.11. A direct proof of $\vdash(A \equiv(A \vee \neg A)) \equiv A$ is also not difficult.) Detaching the left-hand side (using A12, A13, A14, and R1), we conclude $\Gamma \vdash A$.

Theorem 6.7 The orthomodular law does not hold in $\mathcal{A}$.

Proof. This is Theorem 3.27 from [7], and the proof provided there runs as follows. We assume $\mathcal{F}^{\circ}$ contains at least two elementary (primitive) propositions $p_{0}, p_{1}, \ldots$ We pick a valuation $o$ that maps two of them, $A$ and $B$, to distinct nodes $o(A)$ and $o(B)$ of $\mathrm{O} 6$ that are neither 0 nor 1 such that $o(A) \leq o(B)$ [i.e., $o(A)$ and $o(B)$ are on the same side of hexagon O6 in Figure [1. From the structure of O6, we obtain $o(A) \cup o(B)=o(B)$ and $o(A) \cup\left(o(A)^{\prime} \cap(o(A) \cup o(B))\right)=o(A) \cup\left(o(A)^{\prime} \cap o(B)\right)=o(A) \cup 0=o(A)$. Therefore $o(A) \cup o(B) \neq o(A) \cup\left(o(A)^{\prime} \cap(o(A) \cup o(B))\right.$, i.e., $o(A \vee B) \neq$ $o(A \vee(\neg A \wedge(A \vee B)))$. This falsifies $(A \vee B) \approx(A \vee(\neg A \wedge(A \vee B))$. Therefore $a \cup b \neq a \cup\left(a^{\prime} \cap(a \cup b)\right)$, providing a counterexample to the orthomodular law for $\mathcal{F}^{\circ} / \approx$. 
Lemma 6.8 $\mathcal{M}_{\mathcal{A}}=\langle\mathcal{A}, f\rangle$ is a proper WOML model.

Proof. Follows from Lemma 6.5 and Theorem 6.7.

Now we are able to prove the completeness of $\mathcal{Q L}$, i.e., that if a formula $\mathrm{A}$ is a consequence of a set of wffs $\Gamma$ in all WOML-OML models, then $\Gamma \vdash A$. In particular, when $\Gamma=\varnothing$, all valid formulae are provable in $\mathcal{Q} \mathcal{L}$. (Recall from the note below Definition 5.9 that the left-hand side of the metaimplication below is implicitly quantified over all proper WOML models $\mathcal{M}$.)

Theorem 6.9 [Completeness] $\Gamma \vDash_{\mathcal{M}} A \Rightarrow \Gamma \vdash A$.

Proof. $\quad \Gamma \vDash_{\mathcal{M}} A$ means that in all proper WOML models $\mathcal{M}$, if $f(X)=1$ for all $X$ in $\Gamma$, then $f(A)=1$ holds. In particular, it holds for $\mathcal{M}_{\mathcal{A}}=$ $\langle\mathcal{A}, f\rangle$, which is a proper WOML model by Lemma 6.8. Therefore, in the Lindenbaum algebra $\mathcal{A}$, if $f(X)=1$ for all $X$ in $\Gamma$, then $f(A)=1$ holds. By Lemma 6.6, it follows that $\Gamma \vdash A$.

\section{The Completeness of Classical Logic for WDOL-BA Models}

We have to impose the structure of WDOL-BA on the set $\mathcal{F}^{\circ}$ of formulae of $\mathcal{C} \mathcal{L}$. We start with a relation of congruence, i.e., a relation of equivalence compatible with the operations in $\mathcal{C} \mathcal{L}$. We make use of an equivalence relation to establish a correspondence between formulae of $\mathcal{Q} \mathcal{L}$ and formulae of WDOL-BA. The resulting equivalence classes stand for elements of a proper WDOL (i.e., a member of WDOL-BA) and enable the completeness proof of $\mathcal{Q} \mathcal{L}$ by means of WDOL-BA. We will closely follow the procedure outlined in Section 6 and will often implicitly assume that definitions and theorems given in that section for $\mathcal{Q L}$ have a completely analogous form for $\mathcal{C} \mathcal{L}$.

Theorem 7.1 The relation of equivalence $\approx_{\Gamma, \mathcal{C L}}$ or just $\approx$, defined as

$$
\begin{aligned}
A & \approx B \\
& \stackrel{\text { def }}{=} \Gamma \vdash A \equiv_{0} B \&(\forall 7)
\end{aligned}
$$

is a relation of congruence in the algebra $\mathcal{F}$.

Proof. As given in [18]. 
Lemma 7.2 The Lindenbaum algebra $\mathcal{A}=\left\langle\mathcal{F}^{\circ} / \approx, \neg / \approx, \vee / \approx\right\rangle$ is a WDOL, i.e., Eqs. (57)-(60) and Eq. (61) hold for $\neg / \approx$ and $\vee / \approx$ as' and $\cup$ respectively.

Proof. In analogy to Lemma 6.5 and following [18].

Lemma 7.3 In the Lindenbaum algebra $\mathcal{A}$, if $f(X)=1$ for all $X$ in $\Gamma$ implies $f(A)=1$, then $\Gamma \vdash A$.

Proof. As given in [18].

Theorem 7.4 Distributivity does not hold in $\mathcal{A}$.

Proof. $(a \cap(b \cup c))=((a \cap b) \cup(a \cap c))$ fails in O6.

Lemma 7.5 $\mathcal{M}_{\mathcal{A}}=\langle\mathcal{A}, f\rangle$ is a proper WDOL model.

Proof. Follows from Lemma 7.2 and Theorem 7.4 .

Theorem 7.6 [Completeness] $\Gamma \vDash_{\mathcal{M}} A \Rightarrow \Gamma \vdash A$

Proof. Analogous to the proof of Theorem 6.9.

\section{Valuation-Nonmonotonicity}

In Sections 5, 6, and 7 we prove the soundness and completeness of both quantum $(\mathcal{Q L})$ and classical $(\mathcal{C} \mathcal{L})$ standard logic for proper weakly orthomodular (WOML-OML) and weakly distributive (WDOL-BA) ortholattices, respectively. As we stressed in the Introduction and in Section 4, WOML-OML is the class of all those ortholattices (see Definition 2.1) that satisfy Definition 2.8 (WOML) but do not satisfy Definition 2.9. Analogously, WDOL-BA includes all those ortholattices that satisfy Definition 3.5 but do not satisfy Definition 3.2.

The set-theoretical differences WOML $\backslash$ OML (WOML-OMLs) and WDOL $\backslash$ BA (WDOL-BAs) determine valuations that quantum and classical logic can respectively make use of. The set of valuations that can be assigned to logical propositions are simply elements of any of particular lattices, e.g., O6 given in Figure 1. Of course, any standard Boolean valuation set such as, e.g., $\{0,1\}$, i.e., $\{$ TRUE,FALSE $\}$, is then precluded by definition. On the other 
hand, if we decide to use, e.g., $\{0,1\}$-valuation, i.e., two-valued BA as our model, then we cannot use WDOL-BAs valuations any more.

Both WOML-OMLs and WDOL-BAs, on the one hand, and OMLs and BAs, on the other, are models for which we can prove soundness and completeness of quantum and classical logic, respectively. Which ones we will use, i.e., which valuations we will choose, depends on the hardware, i.e., the kind of implementation we adopt. For an implementation of the $\{0,1\}$ valuation, we use today's binary chips; for the $\mathrm{O} 6$ or any other non-Boolean valuation, we might design appropriate chips and circuits in the future. Actually there are certainly many more non-Boolean valuations than the $\mathrm{O} 6$ one, if not infinitely many.

For example, in [11, Th. 3.2] we proved that equation

$(a \equiv b) \cap((b \equiv c) \cup(a \equiv c))=((a \equiv b) \cap(b \equiv c)) \cup((a \equiv b) \cap(a \equiv c))$,

which holds in any OML, does not hold in all WOMLs, since it fails in the Rose-Wilkinson ortholattice in Figure 3 which satisfies the WOML condition Eq. (9).

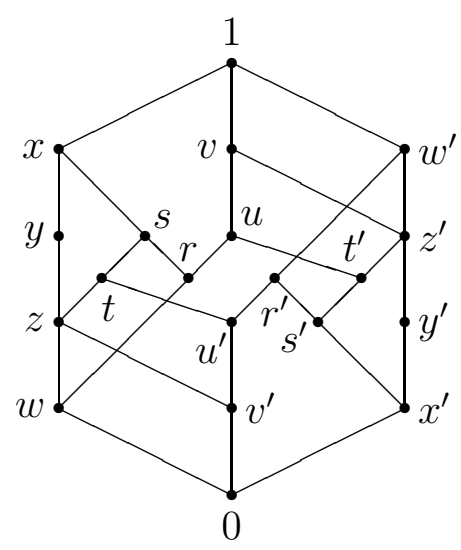

Figure 3: Rose-Wilkinson lattice

If we add Eq. (68) to the WOML conditions, we get a family of latticeslet us call it WOMLi - which is strictly smaller than WOML and strictly larger than OML. One of its valuations is obviously on the O6 lattice but not on the Rose-Wilkinson lattice. In analogy to the way we introduced proper WOMLs in Section 4, we can define WOMLi-OML as the class WOMLi $\backslash$ OML, each member of which is a proper WOMLi. Now the class WOML contains 
both the Rose-Wilkinson and O6 lattices. The class WOMLi-OML will contain $\mathrm{O} 6$ but not the Rose-Wilkinson lattice. The class OML will contain neither Rose-Wilkinson nor O6. A slight modification of the proof of Section 6] (by replacing WOML with WOMLi) shows that quantum logic is complete for WOMLi-OML, and it is also complete for WOMLi itself as follows from the completeness proofs of quantum logic for WOML given in [7, 8].

Alternatively, we can obtain a hierarchy of classes of models for quantum logic by adding conditions to the equations determining the class WOML. Rather than restricting WOML by subtracting OML from it (to obtain WOML-OML), we restrict WOML by adding new conditions (stronger than the WOML law but weaker than the orthomodular law) to its defining equations to obtain smaller equational varieties, in between OML and WOML. We obtain the analogous hierarchy for classical logic by substituting "WDOL" for "WOML," "BA" for "OML," and "distributive" for "orthomodular." For instance, if we start with WOML, we can choose any model from it we wish: O6, Rose-Wilkinson, Beran 7b [20, Fig. 7b], or any other WOML lattice. When we add the condition (68) we can no longer use, e.g., the Rose-Wilkinson lattice/valuation. When we add the orthomodular law, we can no longer use O6 or Rose-Wilkinson or Beran 7b valuations. Thus by adding conditions to the definitions of WOML and WDOL, we change values (valuations) of logical propositions and we call this valuation non-monotonicity. More formally:

Theorem 8.1 Quantum (classical) logic is sound and complete with respect to either the WOML (WDOL) or the OML (BA) model families or any model family which is in between WOML (WDOL) and OML (BA) (such as WOMLi above). Particular WOML, WDOL, OML, BA, WOMLi lattices represent valuation sets for logical propositions. By adding conditions to Definitions 2.1 and 2.8 (WOML), 2.1. and (68) (WOMLi), 2.1 and 3.1 (WDOL), etc. we change the sets of valuations that can be ascribed to propositions. This property of logical propositions getting new sets of values, when we add new conditions to the original definition of lattices to model our logic with, we call valuation-nonmonotonicity

Proof. The soundness and completeness proofs for WOML and WDOL are given by Theorems $29 \& 39$ and $30 \& 47$ of [8] (or by Theorems $3.1 \& 3.29$ and $4.3 \& 4.11$ of [7]), respectively. The soundness and completeness proofs for OML and BA are well known. See, e.g., [17] and [19]. Soundness and completeness proofs for any lattice in between WOML and OML and in between 
WDOL and BA follow from the respective proofs for WOML and OML. For the soundness part of the proof, this is because any such WOMLj or WDOLj $(\mathrm{j}=1,2, \ldots)$ is a WOML or WDOL, respectively. We can obtain a proof that quantum (classical) logic is complete for WOMLj (WDOLj) by rewriting the completeness proof of Section 6 (7) so that the set of mappings to O6 that refines the equivalence relations is replaced by a set of mappings to a lattice that satisfies WOMLj (WDOLj) but violates WOMLj+1 (WDOLj+1), e.g., the Rose-Wilkinson lattice for WOMLj $=$ WOML and WOMLj+1 $=$ WOMLi. The part of the proof that refers to adding conditions is obvious from the very definitions of WOML, WOMLj, OML, WDOL, WDOLj, and BA.

We stress here that we cannot mix up the two alternative ways of choosing valuations (restricting classes and forming set differences vs. valuationnonmonotonicity), because if we added, e.g., the conditions defining OML (BA) to WOML-OML (WDOL-BA), we would simply get empty sets.

\section{Completeness for Smaller Model Subclasses}

The reader familiar with the authors' earlier completeness proofs in [7] will notice that the new proofs here, in Sections 6 and 7, are identical except for the replacement of WOML (WDOL) with WOML-OML (WDOL-BA) in certain places. This yields a stronger result for each logic $(\mathcal{Q L}$ and $\mathcal{C} \mathcal{L})$, i.e., each is complete for a smaller class of models. If a logic is complete for a class of models, it obviously continues to be complete if more models for the logic are added to that class. Thus the earlier completeness results follow immediately from the new ones, since WOML is obtained from WOML-OML by adding back the OML models for $\mathcal{Q L}$ (and analogously WDOL for $\mathcal{C} \mathcal{L}$ ).

The key idea that allowed us to exclude OML from WOML in the $\mathcal{Q} \mathcal{L}$ completeness proof was refinement of the equivalence relation in Theorem 6.2 with the set of mappings $\mathcal{O} 6$. This resulted in smaller equivalence classes, allowing us to construct a Lindenbaum algebra that violated the orthomodular law and is thus a proper WOML.

In fact, the $\mathcal{O} 6$ "trick" is not limited to the use of lattice O6. We can rewrite the completeness proof for e.g. $\mathcal{Q} \mathcal{L}$ using any lattice that is a proper WOML (a WOML but not an OML) in place of O6. This will result in a completeness proof for a different class of models that can be an even smaller subclass of WOML. 
For example, the Rose-Wilkinson lattice of Figure 3 is a proper WOML. If we use it in place of $\mathrm{O6}$, an analogous completeness proof shows that $\mathcal{Q} \mathcal{L}$ is complete for the class WOML $\backslash$ WOMLi, which is strictly smaller than WOML-OML. Since WOML $\backslash$ WOMLi doesn't include O6, this shows that $\mathcal{Q L}$ is complete for a class of models that is not only unrelated to OMLs but is even unrelated to the "natural" OML counterexample O6, which up to now has served as our prototypical WOML example.

As mentioned earlier, for classical logic $\mathcal{C} \mathcal{L}$, we have an even stronger completeness result that it is complete for single WDOL lattices, not just classes of them. For example, it turns out that the Rose-Wilkinson lattice is also a proper WDOL (as well as a proper WOML). Thus the Rose-Wilkinson lattice, by itself, provides a model for which classical logic is sound and complete, showing that the hexagon O6 is not the only "exotic" non-Boolean lattice model for $\mathcal{C} \mathcal{L}$.

\section{Conclusion}

The main result we obtained in the previous sections is that logics can be modelled by disjoint classes of different ortholattices. Classical logic can be modelled by non-distributive lattices and quantum logic by nonorthomodular lattices. These lattices represent different disjoint valuation sets, where the valuation is a mapping from propositions to a lattice. Thus by adding conditions (axioms) to the original definition of an ortholattice we determine classes of lattices that in turn determine valuations that one can ascribe to logical propositions. We call the latter property of logical propositions valuation-nonmonotonicity (see Theorem 8.1). But by considering disjoint classes of lattices we can further restrict valuations we want to use. This can be done as follows.

We considered varieties of classical non-distributive weakly distributive lattice (WDOL, see Definition 3.4) models of classical propositional logic and non-orthomodular weakly orthomodular lattice (WOML, Definition 2.9) models of quantum quantum propositional logics and proved their soundness and completeness for those models (see Theorems [5.10, 5.11, 6.9, and [7.6)

In particular, we considered subclasses of WDOL and WOML that do not contain Boolean algebras (BAs, Definition 3.2) and orthomodular lattices (OMLs, Definition 2.9), respectively, while in Sections 8 and 9 we also considered a possibly infinite sequence of subclasses of WDOL and WOML 
that do not contain lattices WDOLi and WOMLi, respectively, which in turn properly contain BA and OML, and for all of which we have proved the soundness and completeness. We denoted these classes (varieties of WDOL and WMOL) as WDOL-BA, WOML-OML, WDOL-WDOLi, and WMOLWOMLi. The valuations of WOML-OML and OML, of WDOL-BA and BA, of WODL-WODLi and WODLi, of WOML-WOMLi and WOMLi [Eq. (68)], and of WOMLi-OML and OML do not overlap. For instance, valuations from WDOL-BA cannot be numeric $(\{0,1\}$ or $\{$ TRUE,FALSE $\})$ at all since it does not contain the two-valued Boolean algebra.

At the level of logical gates, classical or quantum, with today's technology for computers and artificial intelligence, we can use only bits and qubits, respectively, i.e., only valuations corresponding to $\{0,1\}$ BA and OML, respectively. And when we talk about logics today, we take for granted that they have the latter valuation - $\{$ TRUE,FALSE $\}$ in the case of classical logic and Hasse (Greechie) diagrams in the case of quantum logic [21. This is because a valuation is all we use to implement a logic. In its final application, we do not use a logic as given by its axioms and rules of inferences but instead as given by its models. Actually, logics given only by their axioms and rules of inferences (in Sections 5.1 and 5.2), i.e., without any models and any valuations, cannot be implemented in any hardware at all.

It would be interesting to investigate how other valuations, i.e., various ortholattices, might be implemented in complex circuits. That would provide us with the possibility of controlling essentially different algebraic structures (logical models) implemented into radically different hardware (logic circuits consisting of logic gates) by the same logic as defined by its axioms and rules of inference.

Acknowledgement Supported by the Ministry of Science, Education, and Sport of Croatia through the project No. 082-0982562-3160.

\section{References}

[1] H. Leblanc, Alternatives to Standard First-Order Semantics, in Handbook of Philosophical Logic, edited by D. Gabbay and F. Guenthner, volume I: Elements of Classical Logic, pages 189-274, D. Reidel, Dordrecht, 1983. 
[2] G. Boole, An Investigation of the Laws of Thought, on Which Are Founded the Mathematical Theories of Logic and Probabilities, Walton \& Maberly, London, 1854, Repr. Dover, New York, 1958.

[3] W. V. Quine, Methods of Logic, Routledge \& Kegan Paul, London, 3rd edition, 1970.

[4] M. Pavičić, J.-P. Merlet, and N. D. Megill, Exhaustive Enumeration of Kochen-Specker Vector Systems, The French National Institute for Research in Computer Science and Control Research Reports RR-5388 (2004).

[5] M. Pavičić, J.-P. Merlet, B. D. McKay, and N. D. Megill, KochenSpecker Vectors, J. Phys. A 38, 497-503 (2005), Corrigendum, J. Phys. A 38, 3709 (2005).

[6] M. Pavičić, Quantum Computation and Quantum Communication: Theory and Experiments, Springer, New York, 2005.

[7] M. Pavičić and N. D. Megill, Non-Orthomodular Models for Both Standard Quantum Logic and Standard Classical Logic: Repercussions for Quantum Computers, Helv. Phys. Acta 72, 189-210 (1999).

[8] M. Pavičić and N. D. Megill, Is Quantum Logic a Logic?, in Handbook of Quantum Logic and Quantum Structures, edited by K. Engesser, D. Gabbay, and D. Lehmann, volume Quantum Logic, Elsevier, Amsterdam, 2008.

[9] P. Mittelstaedt, Quantum Logic, Synthese Library; Vol. 18, Reidel, London, 1978.

[10] N. D. Megill and M. Pavičić, Deduction, Ordering, and Operations in Quantum Logic, Found. Phys. 32, 357-378 (2002).

[11] N. D. Megill and M. Pavičić, Equivalences, Identities, Symmetric Differences, and Congruences in Orthomodular Lattices, Int. J. Theor. Phys. 42, 2797-2805 (2003).

[12] G. Kalmbach, Orthomodular Lattices, Academic Press, London, 1983. 
[13] M. Pavičić and N. D. Megill, Binary Orthologic with Modus Ponens Is either Orthomodular or Distributive, Helv. Phys. Acta 71, 610-628 (1998).

[14] M. Pavičić, Identity Rule for Classical and Quantum Theories, Int. J. Theor. Phys. 37, 2099-2103 (1998).

[15] E. Schechter, Classical and Nonclassical Logics: An Introduction to the Mathematics of Propositions, Princeton University Press, Princeton, 2005.

[16] M. Pavičić, Minimal Quantum Logic with Merged Implications, Int. J. Theor. Phys. 26, 845-852 (1987).

[17] G. Kalmbach, Orthomodular Logic, Z. math. Logik Grundl. Math. 20, 395-406 (1974).

[18] M. Pavičić and N. D. Megill, Quantum Logic and Quantum Computation, in Handbook of Quantum Logic and Quantum Structures, edited by K. Engesser, D. Gabbay, and D. Lehmann, volume Quantum Structures, pages 751-787, Elsevier, Amsterdam, 2007.

[19] D. Hilbert and W. Ackermann, Principles of Mathematical Logic, Chelsea, New York, 1950.

[20] L. Beran, Orthomodular Lattices; Algebraic Approach, D. Reidel, Dordrecht, 1985.

[21] B. D. McKay, N. D. Megill, and M. Pavičić, Algorithms for Greechie Diagrams, Int. J. Theor. Phys. 39, 2381-2406 (2000). 